\title{
Ein Beitrag zur mikroskopischen Anatomie der Nebennieren bei Säugethieren.
}

\author{
Von
}

\author{
Dr. med. A. Dostoiewsky
}

aus St. Petersburg.

Hierzu Tafel XIII.

Seit längerer Zeit mit Untersuchungen des Baues derjenigen Gebilde beschäftigt, die man gegenwärtig zu den Blutgefässdrüsen zählt, will ich im nachstehenden die Ergebnisse meiner zweijährigen Untersuchungen über die Nebennieren darstellen. Ein Theil dieser Untersuchungen wurde im histologischen Laboratorium zu St. Petersburg ausgefuihrt, ein anderer Theil im Berliner anatomischen Institut im Laufe des Jahres 1885.

Macht man einen Schnitt aus der Nebenniere irgend eines Thieres, so nimmt man schon mit blossem Auge wahr, dass das Organ aus zwei verschiedenen Substanzen zusammengesetzt ist einer centralen und einer peripheren - oder mit anderen Worten, aus einer Rinden- und einer Marksubstanz. Die erstere umgiebt in Gestalt eines dicken weissen oder gelblichen Ringes von allen Seiten die röthliche Marksubstanz. Die gegenseitige Lage dieser beiden Theile ist nicht immer die nämliche. In manchen Fällen sieht man auf Querschnitten, dass die Grenze zwischen der Rinden- und der Marksubstanz parallel mit der äusseren Oberfläche des Organs verläuft, dass mithin im Allgemeinen die Form der Marksubstanz die Form der ganzen Drüse wiederholt; in anderen Fällen erscheint die Grenze zwischen Rinden- und Marksubstanz in Gestalt einer gebrochenen Linie mit verschiedenen Biegungen nach der einen und der anderen Seite hin: zuweilen dringt die Marksubstanz mit einem Fortsatz dicht bis an die Kapsel heran, andererseits sieht man oft Bezirke von Rindensubstanz inmitten der Marksubstanz. 
Ein Beitrag zur mikroskop. Anatomie der Nebennieren bei Säugethieren. 273

\section{Die Rindensubstanz.}

In Bezug auf die Struktur der Rindensubstanz existiren zwei vollkommen entgegengesetzte Ansichten. Ecker $\left.{ }^{2}\right)^{3}$ ), der die Nebennieren für wahre Driisen hält, behauptet, dass sie alle Merkmale und Eigenthümlichkeiten besitzen, die nach den damaligen Anschauungen den wahren Drüsen zukommen sollten. Die drüsigen Elemente, welche aus Kernen und Zellen besteben und in einer feinkörnigen Zwisehensubstanz eingebettet sind, werden von einer besonderen Membrana propria eingeschlossen und somit ist die ganze Rindensubstanz von einem Complex länglicher Schläuche gebildet, die einander parallel gelagert sind und senkrecht von der Kapsel zur Marksubstanz hinziehen. Mit Hilfe von Kali und von Ammoniak konnte er sich jedoch iiberzeugen, dass das Bild der langen Schlïuche auf einer genauen Auseinanderlagerung zablreicher kleinerer, zumeist ovalen Bläschen beruht. Demnach ist nach der Ansicht Ecker's der wesentliche Bestandtheil der Rindensubstanz ein Bläschen mit strukturloser Hülle und einem Inhalt. Einer ganz anderen Ansicht huldigt Kölliker ${ }^{4}$ ). Nach seiner Meinung besteht der grösste Theil der Rindensubstanz nicht aus Drüsenbläschen. Die letzteren kommen nur selten in den innersten Partien der Rindensubstanz vor, an der Grenze derselben mit der Marksubstanz. Der grösste Theil der Rindensubstanz besteht aus Parenchymelementen, die frei in einem bindegewebigen Stroma liegen und nicht von einer Membrana propria umschlossen sind. Die Autoren, welche später über den Bau der Rindensubstanz schrieben, schlossen sich im Grossen und Ganzen entweder Eckeran, wie z. B. Frey, Hassal, Gerlach ${ }^{5}$ ), Luschka ${ }^{6}$ ), Leydig ${ }^{7}$ ), Grandry ${ }^{8}$ ) a. A., oder an Kölliker, wie z. B. Arnold ${ }^{9}$ ), Moers ${ }^{10}$ ), Brunn ${ }^{11}$ ) u. A. Die Forscher der letztern Kategorie erkennen zwar im Allgemeinen an, dass die Rindensubstanz aus Bindegewebe mit darin eingelagerten Parenchymelementen zusammengesetzt sei, stimmen aber sowohl in Bezug auf den Bau als auch auf die Anordnung der genannten Theile mit einander nicht überein. Nach der Ansicht von Kölliker ist die ganze Rindensubstanz einförmig gebaut: in Bindegewebemaschen verschiedener Grösse liegt je eine Anzahl Zellen. Moers und Joesten ${ }^{12}$ ) beschrieben in der Rindensubstanz zwei Schichten: in den änsseren Partien bildet das Bindegewebe grosse runde Maschen 
und in den inneren zieht dasselbe senkrecht unter Bildung länglicher Fächer. Arnold unterscheidet drei Schichten: eine Zona glomerulosa, eine Zona fasciculata und eine Zona reticularis. Diese Eintheilung wird durch die Anordnung der gröberen Bindegewebsbalken bedingt, ausserdem ist die ganze Rindensubstanz nach A rnold von einem feinsten Reticulum durchsetzt. Eberth ${ }^{13}$ ) beschreibt mit Moers beim Rinde ein feinstes Netzwerk nur in den inneren Partien der Rindensubstanz. von Brunn unterscheidet ebenfalls drei Schichten, aber er fand ein Reticulum nur in den zwei inneren Schichten Arnold's. Indem Rauber ${ }^{14}$ ) die Nebennieren bei einer Reihe von Thieren sowie beim Menschen untersuchte, gelangte er za dem Schlusse, dass in der Rindensubstanz das zum Theil faserige, zum Theil lamellöse Bindegewebe, von der Kapsel des Organs ausgehend, allseitig geschlossene Räume bildet. Unmittelbar an der Kapsel sind diese Räume ziemlich gross, rundlich, weiter in der Richtung nach innen verlängern sie sich und werden gegen die Mitte der Rindensubstanz so klein, dass in ihnen nur je eine Zelle zu liegen kommt. Gottschau ${ }^{15}$ ) theilt mit Arnold die Rindensubstanz in drei Schichten, aber er nimmt als Grundlage dieser Eintheilung nicht die Art und Weise der Anordnung des Bindegewebes an, sondern die Eigenschaften der Parenchymzellen, d. h. ihre Grösse, Form und chemischen Charakter. In der Mitte zwischen den Ansichten, als deren Vertreter einerseits Ecker und andererseits Kölliker erschienen, steht die Ansicht von Henle ${ }^{16}$ ). In allen seinen Arbeiten spricht er sich in dem Sinne aus, dass die Zellen entweder frei im Bindegewebsstroma liegen oder in Schläuchen angeordnet seien. Dieser Umstand hängt von der Form und den Bestandtheilen der Zellen ab, unter welchen Henle in den äussersten Gliedern zwei Arten von Zellen unterscheidet, die unter sich durch $Z$ wischenformen verbunden sind. Zellen mit hellem Protoplasma liegen frei, Zellen mit fettigem Inhalt sind in Drüsenschläuche eingeschlossen. Ein feinstes Reticulum stellt Henle vollkommen in Abrede und hält dasselbe für ein Kunstprodukt, hervorgebracht durch Behandlung des Organs mit Chromsäure.

Ich untersuchte die Nebennieren - abgesehen vom Menschen beim Rinde, beim Kalbe, beim Pferde, beim Schafe, beim Schweine, beim Hunde, bei der Katze, beim Kaninchen, beim Meerschweinchen und bei der Ratte. Die Drüsen wurden sowohl erwachsenen 
als jungen Thieren entnommen, in verschiedenen Stadien der Verdauung, in verschiedenen Jahreszeiten, sowohl trächtigen Weibchen, als nicht trächtigen. Selbst bei oberflächlicher Untersuchung des Baues der Nebennieren kann man sich überzeugen, dass sie sich nicht bei allen Thieren auf einen Typus zurückfuhren lassen. Wir wollen mit der Beschreibung der Rindensubstanz beim Rinde, beim Schweine und beim Pferde, die in ihrem Bau Vieles gemein haben, beginnen.

Die Kapsel der Nebennieren ist mit dem Parenchym derselben fest verwachsen und lässt sich nicht auf grössere Strecken frei ablösen. Dieser Zusammenhang wird durch ein System ziemlich dicker Bindegewebsbalken vermittelt, die von der Kapsel ausgehen und in senkrechter Richtung nach dem Centrum des Organs sich begeben. Einige von diesen Balken und zwar die, welche die grossen Gefässe und die Nervenstämme führen, kommen bis an die Marksubstanz heran und dringen in dieselbe ein, andere dagegen, die minder dicken, treten in die Rindensubstanz auf eine grössere oder geringere Entfernung ein und zerfallen in die sie zusammensetzenden Fasern. Studirt man den Bau des Stromas der Rindensubstanz bei den genannten Thieren, so kann man sich überzeugen, dass dasselbe in verschiedenen Abschnitten nicht gleich beschaffen ist. Von der Kapsel gehen ausser den erwähnten groben Bindegewebsbalken kleinere Fortsätze ab, die das Aussehen von Lamellen oder Fasern haben und an der Kapsel Fächer oder Räume verschiedener Grösse bilden. Beim Pferde besitzen dieselben eine längliche Gestalt und sind rechtwinklig zur Kapsel angeordnet; beim Rinde, beim Schweine und beim Schafe erscheinen sie länglich, oval oder rund. Aehnliche Bindegewebsfortsätze gehen auch von den groben Balken ab und bilden um dieselben herum ähnliche Räume wie dicht an der Kapsel. Mithin ist diese weitmaschige Bindegewebsschicht an der inneren Seite der ganzen Kapsel angeordnet, steigt gemeinsam mit den Balken in's Innere der Rindensubstanz herab and umgiebt die letzteren allseitig in Gestalt einer Scheide. Indem die Balken, wie bereits erwähnt, verschieden tief in die Rindensubstanz eindringen, werden sie auch auf dieselbe Entfernung von der Schicht weitmaschigen Bindegewebes begleitet. Wie wir später zeigen werden, sind in diesen weiten Maschen Parenchymelemente gelagert, die sich von den übrigen Zellen der Rindensubstanz unterscheiden. Auf diese Weise 
kann man beim Pferde und beim Rinde nicht selten an der Grenze gegen die Marksubstanz und mitunter auch in der letzteren selbst auf Bezirke stossen, die ganz ähnlich beschaffen sind, d. h. ein ganz ähnliches Stroma und Parcnchymelement haben wie unmittelbar an der Kapsel. Demnach besizt diese weitmaschige Bindegewebsschicht auf Verticalschnitten das Aussehen von Arcaden, wobei als äussere Grenzen oder Seiten die Balken erscheinen und an der Peripherie - die Kapsel.

Nachdem die Bindegewebsbündel die weitmaschige Schicht gebildet haben, zerfallen sie in feinste Fäserchen, die sich in versehiedener Weise unter einander vereinigen, am ein feinstes Reticulum berzustellen. Die Mehrzahl der Bündel verläuft vor ilırem definitiven Zerfall in senkrechter Richtung und in der Richtung nach der Marksubstanz. In den innersten Partien der Rindensubstanz betheiligen sich an der Bildung des Reticulum auch manche von den dicken Balken. Dies geschieht in der Weise, dass ein Balken an einer gewissen Stelle plötzlich in eine Menge feinster Fasern zerfällt. In anderen Fällen geht die Zerfaserung' allmählich vor sich. Zuweilen sieht man, dass ein Balken bald nach seinem Abgang von der Kapsel sich gabelförmig theilt, eine Gruppe von Zellen umfasst, sich wiederum vereinigt und erst dann, nachdem eine gewisse Strecke zurückgelegt ist, definitiv zerfällt. Anlangend den Bau dieses Stroma, so sind die dicken Balken ganz ähnlich beschaffen wie die Kapsel. Das Bindegewebe, welches die weitmaschige Schicht bildet, hat die Gestalt von Lamellen resp. Fasern mit darin gelegenen Bindegewebszellen; die Fasern, welche das Netzwerk zusammensetzen, sind ausserordentlich dünn und homogen, so dass an Schichten, in welchen die Parenchymelemente in loco geblieben sind, dieses Netz sogar schwer zu sehen ist. Wenn man aber die Zellen mittelst eines Pinsels entfernt, so lässt sich dasselbe in ziemlich bedeutender Ausdehnung präpariren und man kann sich von seiner Existenz überzeugen. Die beigegebene Figur 1 stellt das feinste Netz aus der Rindensubstanz des Schweines dar. Stellenweise zeigen die Fasern Verdickungen, welche sich tief mit Hämatoxylin und mit Pikrocarmin färben: das sind Kerne von Bindegewebszellen. Oben wurde angeführt, dass Henle die Existenz eines Reticulum leugnet; nach seiner Meinung soll dasselbe ein Kunstprodukt sein, hervorgebracht durch Behandlung des Präparates mit Chromsäure und ihren Salzen. Allein ab- 
gesehen davon, dass man sich von der Existenz eines Reticulum an in Alkohol gehärteten Präparaten zu tüberzengen vermag; kann man dasselbe auch an Schnitten sehen aus dem gefrorenen, vollkommen frischen Organ. Ausserdem sprechen für die Existenz eines solchen Netzes die Kerne, welche sich mit Hämatoxylin and mit Pikocarmin färben.

Die Parenchymelemente, welche in dem soeben beschriebenen Stroma gelagert sind, lassen sich in zwei Sorten eintheilen. Die einen liegen unmittelbar an der Kapsel und um die dicken Bündel herum, d. h. in der weitmaschigen Schicht des Bindegewebes, die andern ordnei sich in der ganzen übrigen Partie der Rindensubstanz an. Diese beiden Sorten von Zellen sind scharf von einander unterschieden. In den langen, senkrecht zur Kapsel gelegenen Räumen der Rindensubstanz des Pferdes beobachtet man die besonders durch v. Brunn beschriebenen, länglichen, spindelförmigen Zellen mit länglichem Kern. Ihre Länge erreicht $0,04-0,05 \mathrm{~mm}$. Sie ordnen sich senkrecht $\mathrm{zu}$ den Bindegewebsfasern an und liegen dicht beieinander. Zwischen zwei Balken befindet sich entweder eine Reihe von Zellen, wobei die letzteren mit ihren Enden die Balken beiderseits berïhren, oder es befinden sich zwei Reihen von Zellen, wobei die schmalen Enden der Zellen der einen Reihe sich in die Zwischenräume zwischen den Zellen der anderen Reihe hineinschieben. Manchmal gehen solche Reihen von zwischen den Bindegewebsfasern angeordneten Zellen dicht unterhalb der Kapsel hufeisenförmig in einander über. Die Breite dieser Schicht bein Pferde oder mit anderen Worten die Länge der Fächer erreicht $0,5 \mathrm{~mm}$. Beim Rinde und beim Schweine sind die Maschen der weitmaschigen Schicht kleiner als beim Pferde und besitzen in der Mehrzahl der Fälle eine runde oder ovale Gestalt. Angeordnet sind dieselben unterhalb der Kapsel in mehreren Reihen, so dass also diese zweite Schicht nahezu von derselben Breite ist wie beim Pferde. Die Zellen sind hier gleichfalls spindelförmig und cylindrisch, es kommen jedoch häufig runde und polygonale vor. Der Kern nimmt den grössten Theil der Zelle ein. Es finden sich mitunter ziemlich kleine Maschen vor, so dass man auf Schnitten 10-20 Zellen in denselben zu zählen vermag. Das Protoplasma der Zellen ist bei allen genannten Thieren feinkörnig und enthält keine Fettkïrnchen. Genau solche Zellen liegen in den grossen Bindegewebsmaschen um die grossen Balken berum. Indem die Reihen 
der spindelförmigen Zellen diese Balken von allen Seiten auskleiden, steigen sie beim Pferde und beim Rinde mitunter ziemlich tief herab und dringen auch in die Marksnbstanz hinein. Das von J. Arnold beschriebene feinste Reticulum, welches diese grossen Bindegewebsmaschen durchsetzen sollte, existirt nicht. Einen Uebergang von Fortsätzen der Parenchymzellen in das umgebende Bindegewebe, wie es v. Brunn beschreibt, konnte ich ebenfalls nicht beobachten.

Gegen einen solchen Zusammenhang der Zellen mil dem Stroma spricht schon der Umstand, dass die Zellen sehr lose in den Maschen sitzen und leicht aus denselben heransfallen, so dass man selbst bei der allersorgfältigsten Herstellung des Präparats unter Umständen nur das bindegewebige Stroma zu Gesicht bekommt. Sehr häufig ist man in der Lage zu beobachten, dass die Zellen in den Fällen, wo sie erhalten sind, in der Mitte einer Masche liegen zu einem Häufchen angeordnet, und dass zwischen Ien letzteren und den Maschenwandungen ein freier Raum iubrig bleibt.

In der gesammten tibrigen Ausdehnung der Rindensubstanz ist die Form und Anordnung der Zellen gemäss der besonderen Beschaffenheit des Stroma vollkommen abweichend von dem soeben Beschriebenen. Hier sind namentlich bei allen Thieren die Zellen in langen Zügen oder Reihen angeordnet und liegen frei in den Maschen des oben beschriebenen engmaschigen Netzes. Diese Maschen sind so klein, dass in jeder von ihnen nur je eine, seltener mehrere Zellen liegen. Die Anordnung der Zellen in Reihen wird durch den oben beschriebenen Verlauf der dicken Bindegewebsbündel bedingt. In jedem Zuge befindet sich entweder eine Reihe von Zellen oder mehrere Reihen. Sie haben scharfe Contouren in Gestalt von feinen Säumen mit stellenweise darein eingebetteten Kernen. Dieser Umstand gab auch zu der Vermuthung Anlass, dass die Zellen in besondere Schläuche oder Bläschen eingeschlossen seien. Thatsächlich ist der unmittelbar einer Zellenreihe anliegende feine Saum ein Ausdruck der Wand von Capillaren, die zwischen den Zellenreihen in derselben Richtung mit diesen verlaufen.

Die Form und die chemischen Eigenschaften der Zellen dieses Abschnittes der Rindensubstanz sind sehr mannigfaltig bei verschiedenen Tbieren sowohl als bei einzelnen Individuen. Beim 
Rinde, beim Schweine und Schafe besitzen die Zellen in der Mehrzahl der Fälle eine polygonale Form. Sie sind viel grösser als die Zellen der peripheren Sehicht. Sie werden in der Richtung zur Marksubstanz allmählich kleiner, obgleich nicht bedeutend. Beim Rinde erreichen einzelne Zellen unter Umständen eine sehr beträchtliche Grösse. Die Zellen enthalten runde, scharf cortourirte bläschenförmige Kerne mit deutlich sichtbaren Kernkörperchen. Manche Zellen enthalten je zwei Kerne. Das Protoplasma der Kerne ist homogen and führt für gewöhnlich bei den genannten Thieren weder Fettkörner noch irgend welche andere Körner. Beim Pferde haben mitunter die Zellen in ein und derselben Drüse eine verschiedene Struktur. Stellenweise ordnen sich dieselben in regelmässigen Reibeu an, sind gut contourirt, enthalten einen Kern mit deutlich sichtbarem Kernkörperchen. Das Protoplasma ist feinkörnig. In anderen Fällen, mitunter in derselben Drüse und selbst neben den soeben beschriebenen Zellen, ordnen sich Complexe von Zellen an, die in regressiver Metamorphose begriffen zu sein scheinen; sie werden kleiner und in ihrem Protoplasma zeigen sich von allen Seiten Einkerbungen, so dass die Zellen eine sternförmige Gestalt annebmen. Eine geringe Menge Protoplasma bleibt nur um den Kern herum, die Zellen grenzen sich nicht deutlich von einander ab und scheinen mit ihren Fortsätzen zu verschmelzen, so dass die Rindensubstanz auf Schnitten dieser Stellen gleiehsam ein feinstes Gespinnst darbietet. Bei der Beschreibung von sternförmigen Zellen mit Fortsätzen hatte v. Brunn wahrscheinlich diese Zellen im Auge. Entweder ist der Uebergang von den oben beschriebenen gut contourirten Zellen zu den zuletzt genannten ein allmählicher und durch Uebergangsformen vermittelt, oder beide Arten sind scharf von einander abgegrenzt, so dass neben gut contourirten Zellen geschrumpfte liegen.

Der oben dargestellte Bau des Stroma und der Parenchymelemente wird beim Pferde, Rinde, Schweine und Schafe beobachtet: hieran schliesst sich auch die Struktur der Rindensubstanz beim Hunde an. Am meisten nähert sie sich dem Bau in der Nebenniere des Pferdes. Bei den anderen Thieren ist das Stroma etwas abweichend beschaffen. So ist z. B. bei der Katze, beim Kaninchen, beim Meerschweinchen, bei der Ratte das Bindegewebe sehr schwach entwickelt und seine Vertheilung fast dieselbe in allen Theilen der Rindensubstanz. Namentlich lässt sich hier die sog. 
weitmaschige Schicht beinahe gar nicht constatiren. Es gehen zarte Fasern in radiürer Richtung von der Kapsel aus nach innen, verbinden sich unter einander durch zahlreiche quer verlaufende Fasern und bilden ein Netz, in welchem eben die Parenchymelemente eingeschlossen sind. Andererseits beobachtet man beim erwachsenen Menschen eine besondere Entwicklung des Bindegewebes, dabei zieht dasselbe in einer grösseren Partie der Rindensubstanz in Gestalt dicker Bündel, die grosse Maschen entstehen lassen; der Zerfall in ein engmaschiges Netzwerk wird in den innersten Theilen der Rindensubstanz wahrgenommen. Die Zellen ordnen sich beim Kaninchen, bei der Ratte, bei der Katze und beim Meerschweinchen dem Bau des Stroma gemäss gleichförmig über die ganze Rindensubstanz in langen Reihen an, besitzen jedoch nicht in allen Theilen dieselbe Form und dieselben chemischen Eigenschaften. Dicht unterhalb der Kapsel sind sie von geringer Grösse, ihr Protoplasma ist homogen, und sie enthalten je einen grossen Kern. Die Form der Zellen ist meist eine polygonale. Beim Kaninchen kann man Fälle beobachten, wo zwischen zwei Balken eine Reihe ron Zellen Platz findet, die dicht bei einander liegend eine cylindrische Gestalt aufweisen. Spindelförmige Zellen, wie man sie beim Pferde und beim Hunde sehen kann, sind hier nicht vorhanden. In der Richtung nach innen ändert sich die Form der Zellen und ihrer Eigenschaften: nach und nach nehmen sie an Umfang $z a$, und in ibrem Protoplasma erscheinen besondere Körner. Ihre bedeutendste Grösse haben die Zellen in der Mitte der Rindensubstanz. Nach der Marksubstanz zu werden sie viel kleiner.

Ein besonderes Interesse gewinnen diese Zellen in der Hinsicht, dass sie eig en th ï m] ic be Körner enthalten, deren alle Autoren gedenken. Die meisten Körner sind in den Zellen der Rindensubstanz bei der Katze, beim Kaninchen und beim Meerschweinchen vorbanden. An frischen Zupfpräparaten erscheinen dieselben in so enormer Menge, dass sie den Kern vollkommen verdecken. Sie fallen sebr leicht aus den Zellen heraus und schwimmen in der umgebenden Fliissigkeit umber. Die Mehrzahl der Autoren ist geneigt, diese Körner als Fettkörner anzusprechen, allein ihre fettige Natur wird schon von v. Brunn geleugnet. In der That geben sie keine Fettreactionen, werden beispielsweise durch Ueberosmiumsäure fast gar nicht gefärbt, lösen sich jedoch in Aether. Die Vertheilnng der Körner ist entweder eine gleichmïssige iiber 
Ein Beitrag zur mikroskop. Anatomie der Nebennieren bei Säugethieren 281

alle Zellen der Rindensubstanz, wie man dies beim Meerschweinchen and beim Kaninchen beobachten kann, oder, wie man es bei der Katze sieht, erfüllen solche Körner eine gewisse Anzahl von Zellen, ohne dass Uebergangsformen vorkommen, indem neben körnigen Zellen vollständig homogene liegen. Beim Einschluss von Schnitten aus der Rindensubstanz der genannten Thiere in Canadabalsam werden diese Körner unsichtbar infolge ihrer Lösung und Aufhellung im Balsam, und die Zelle bietet ein Fachwerk dar. Dieses Fachwerk oder Gitterwerk ist also nicht der Ausdruck der Struktur des Protoplasmas der Zellen und darf nicht mit dem Fachwerk der Knorpelzellen z. B. oder Leberzellen verglichen werden. Denn in den zuletzt genannten Zellen bilden Fäden thatsächlich einen Theil der Struktur des Protoplasmas, in den Zellen der Nebennieren dagegen ist, wie in den Zellen der Talgdrusen, die fachwerkartige Anordnung des Protoplasmas selbst bedingt durch Einlagerung anders gearteter Körner, die sich nachträglich gelöst baben. Natïrlich schliesst dies nicht die Möglichkeit aus, dass das Protoplasma selbst einerseits, wie man es heut zu Tage für jedes Protoplasma annimmt, ans Fäden und Interfilarmasse zusammengesetzt sei.

Fasst man alles, wie oben geschildert, wieder zusammen, so kann man zu folgenden Schlussen gelangen: Die Rindensubstanz besteht aus einem Stroma mit in dasselbe eingebetteten Parenchymelementen. Nimmt man als Grundlage der Eintheilung, wie es J. Arnold thut, die Anordnung des Bindegewebes, so lässt sich die gesammte Rindensubstanz in zwei Abschnitte zerlegen - einen weitmaschigen und einen engmaschigen. Die in diesen Abschnitten eingebetteten Parenchymelemente sind ebenfalls scharf von einander unterschieden. Diese Abschnitte liegen bei vielen Thieren nicht in Schichten einer tiber dem anderen. Der Bau der innersten Partie der Rindensubstanz, die J. Arnold als Zona reticularis bezeichnet, ist nicht in dem Maasse vom Bau der mittleren Partie abweichend, dass die Aufstellung einer besonderen Schicht geboten wäre. Mithin ist die von J. Arnold vorgeschlagene Theilung der Rindensubstanz in drei über einanderliegende Schichten nicht für alle Thiere anwendbar.

Im inneren Drittel der Rindensubstanz kommen bei manchen Thieren eigenthümliche Bildungen vor, die ein besonderes Interesse verdienen. Es wurde oben angegeben, dass Kölliker in den 
inneren Partien der Rindensubstanz von ihm sogenannte „Schläuche" mit fettigem Inhalte fand. v. Brunn scheint ebenfalls ähnliche Bildungen gesehen zu haben. Henle fand, wie bereits oben erwähnt, "Schläuche" mit Fettzellen in allen Abschnitten der Rindensubstanz. Von anderen Autoren weist Niemand auf ähnliche Gebilde hin. Rauber fasst solche Bilder als pathologische auf. Indessen sind diese Gebilde bei manchen Thieren unzweifelhaft vorhanden und befinden sich nicht bloss an der Grenze der Marksubstanz, sondern auch in verschiedenen Abschnitten derselben. Am häufigsten kann man sie von grossen Thieren beim Pferde und von kleineren beim Kaninchen und bei der Katze beobachten. Beim Pferde kommen in den inneren Partien ber Rindensubstanz sowohl einzelne mit Fetttröpfchen gefüllte Zellen vor, als auch ganze Complexe solcher Zellen, eingeschlossen in eine gemeinsame umhüllende Kapsel. In manchen dieser Kapseln, namentlich in denen, in welchen noch nicht viel Fett vorhanden ist, sind die Grenzen der Zellen sehr gut sichtbar; die Zellen selbst erscheinen im Vergleiche mit den übrigen Zellen dieses Abschnittes vergrössert und enthalten je einen kleinen, gewöhnlich seitlich liegenden Kern. In anderen Kapselräumen werden die Contouren der Zellen minder deutlich sichtbar; zuerst verschwinden dieselben im Centrum der Räume, dann auch an der Peripherie. In den weiteren Stadien der fettigen Infiltration sind die Zellen schon nicht mehr von einander abgegrenzt und fliessen zu einer grobkörnigen Fettmasse zusammen, in der mit Hämatoxylin sich färbende Kerne zerstreut liegen. Solche eingekapselte Zellengruppen in verschiedenen Stadien der fettigen Infiltration bilden beim Pferde eine Schicht an der Grenze zwischen Rinden- und Marksubstanz. Die Zellenhaufen sind von einer bindegewebigen Wandschicht begrenzt, eine sogenannte Membrana propria fehltjedoch. Diese Schicht fetthaltiger Zellen ist an Präparaten aus Müller'scher Flïssigkeit schon mit blossem Auge als weisser Streifen sichtbar. Unter Umständen erreichen iibrigens ähnliche Zellengruppen die Mitte der Rindensubstanz und dringen andererseits in die äusseren Abschnitte der Marksubstanz hinein. Beim Kaninchen zeigen dieselben eine geringere Grösse und liegen nicht so nahe aneinander, um eine Schicht bilden zu können. Sie liegen vielmehr zerstreut in der inneren Hälfte der Rindensubstanz und drängen sich mitunter in die Marksubstanz vor. Die fetthaltigen Zellen sind beim Kaninchen immer gut contourirt und fliessen niemals zu 
einer einzigen Masse zusammen. Auf den ersten Blick erinnern die letzteren auffallend an die Talgdrïsen der Haut.

Fig. 3 und 4 stellen solche Zellengruppen aus den Nebennieren des Pferdes und des Kaninchens dar.

\section{Die Marksubstanz.}

Die Marksubstanz ist complieirter gebaut als die Rindensubstanz, und in Folge dessen kommen in den Schilderungen ihrer Struktur noch mehr Widersprüche vor. Die älteren Forscher beschrieben als Parenchym der Marksubstanz ein feinkörniges Plasma mit darin eingebetteten freien Kernen. Durch spätere Untersuchungen wurde festgestellt, dass die letzten Elemente dẹr Marksubstanz genau wie die der Rindensubstanz Zellen sind. In den Beschreibungen ibrer Natur und Anordnung bestehen aber erhebliche Differenzen. Die einen wie Leydig, Luschka, Kölliker, beschreiben alle Zellen der Marksubstanz als identisch mit Nervenzellen. Andere, wie Joesten und Henle leugnen die nervöse Natur der genannten Zellen und behaupten, dass dieselben in besondere Bläschen oder Schläuche mit eigener Membran eingeschlossen seien. Die Mehrzahl der Autoren hebt aber hervor, dass die Marksubstanz ans einem bindegewebigen Stroma bestehe, das ein Netz bilde, in dessen Maschen eben die zelligen Elemente eingebettet seien. In der Beschreibung des bindegewebigen Stroma stimmen die Autoren mehr oder minder überein. Arnold und v. Brunn beschrieben ausser groben Maschen noch ein feinstes Reticulum, dessen Fasern jede Zelle für sich umgrenzen. Andere Forscher stellen die Existenz eines solchen Reticulum in Abrede. Manche nehmen an, dass die Differenz zwischen Mark- und Rindensubstanz unbedeutend sei. So behauptet z. B. Moers, die Rindensubstanz sowohl als die Marksubstanz werden aus gleichen Elementen zusammengesetzt, ihr Verhalten gegen Reagentien sei ebenfalls nahezu gleich. Das zuerst von Henle beobachtete Vermögen der Zellen der Marksubstanz, sich in Lösungen der Chromsäure und ihrer Salze zu färben wird von allen späteren Autoren bestätigt. Ausser Stroma und specifischen Zellen, die mit Nervenzellen nichts gemein baben und ihnen gar nicht ähnlich sind, entbält die Marksubstanz ein Netz von Nervenfasern, unzweifelhafte Nervenzellen, ein Netz eigenthümlicher Gefässe und schliesslich 
mehr oder minder ausgedehnte Bezirke von Elementen, die Holm als zweifelhafte Nervenzellen bezeichnet hat.

Zunächst will ich das Stroma und die specifischen Zellen der Marksubstanz, d. h. diejenigen Zellen, welche in Lösungen der Chromsäure und ihren Salzen eine braune Farbe annehmen, beschreiben. Macht man einen Querschnitt durch die ganze Marksubstanz einer Nebenniere des Schafes, des Schweines oder des Rindes, so kann man im Centrum eines solchen Schnittes ein quer durchschnittenes grosses Gefäss - die v. centralis, welche das ganze Organ der Länge nach durchzieht und infolge dessen auf allen Querschnitten getroffen wird, wahrnehmen. Unter dem Mikroskop sieht man, dass in dieses Gefäss zahlreiche kleinere Gefässe mlinden, und dass von seiner Adventitia Bindegewebslamellen abgehen, die in radiärer Richtung nach der Rindensubstanz ziehen. Das Bindegewebe in der Nähe der $v$. centralis ist gut entwickelt und besteht aus ziemlich dicken mannichfaltig untereinander sich durchflechtenden Bündeln. In diesem Bindegewebe liegen einzeln die grossen Parenchymzellen. Die letzteren sind von einander isolirt, und den Hauptbestandtheil dieses Abschnittes der Marksubstanz, der unmittelbar an die v. centralis sich anschliesst, stellt das Bindegewebe dar. Mit der Entfernung von der v. centralis zerfällt das Bindegewebe in kleinere Bündel, die sich unter einander durchflechten und dadurch ein Netz mit Maschen verschiedener Form und Grösse bilden. Nach dem Centrum zu sind diese Maschen meist rund und enthalten je 2-5 Zellen. Weiter nach der Peripherie hin werden die Maschen immer grösser, ziehen sich in die Länge und haben auf Durehschnitten die Gestalt langer Schläuche. Mit der Vergrösserung der Maschen wächst auch die Zahl der in ihnen enthaltenen Zellen. Gleichzeitig wird, wie bereits erwähnt, das Bindegewebe immer zarter und ist in den peripheren Partien der Marksubstanz fast gar nicht zu sehen. Das Bild zeigt lange Reihen von Zellen, geschieden durch leere Räume, welche Durchschnitte von Gefässen repräsentiren. Anlangend die Form der Zellen, so ist dieselbe verschieden in verschiedenen Abschnitten der Marksubstanz. In den centralen Partien, wo die Zellen meist einzeln liegen, ist die Form derselhen ausserordentlich mannigfaltig: rund, oval, polygonal u. s. w. Niemals sind jedoch an den Zellen Fortsätze wahrzunehmen, wie es v. Brunn angiebt. Das Protoplasma der Zellen erscheint hier 
feinkörnig. Je weiter nach der Peripherie hin, um so mehr ordnen sich die Zellen in Reihen an, wobei auch ihre Gestalt sich ändert; sie liegen dicht bei einander, besitzen eine cylindrische Form und stehen senkrecht zu dem sie umgebenden Bindegewebe, so dass sie das Aussehen eines wirklichen Cylinderepithels erhalten. Studirt man diese Reihen genauer, so findet man, dass nicht alle Zellen gleich sind: zwischen den oben beschriebenen cylindrischen Zellen, die in Chromsäure und ibren Salzen eine braune Färbung annehmen, liegen runde oder ovale bläschenförmige Zellen mit je einem kleinen excentrisch angeordneten Kerne, die sich sebr schwach in Müller'scher Flüssigkeit färben. Mitunter ist die Gestalt solcher Zellen eine becherförmige und in diesem Falle gewinnen die Reihen cylindrischer Zellen mit stellenweise eingeschalteten hellen becherförmigen Zellen eine entfernte Aehnlichkeit mit dem Darmepithel mit seinen Becherzellen. Diese Zellen sind sehr vergänglicher Natur und gehen leicht zu Grunde: in solchem Falle sieht man auf Schnitten zwischen den cylindrischen Zellen scharf begrenzte leere Räume, deren Form der zu Grunde gegangenen Zelle entspricht. In den äusseren Theilen der Marksubstanz sind die bläschenförmigen Zellen wenig zahlreich; nach dem Centrum hin nehmen sie an Menge zu. Die beigegebene Figur 5 stellt eine Reihe cylindrischer Zellen mit den dazwischen eingeschalteten bläschenförmigen Zellen aus der Marksubstanz des Rindes dar. Die Reihen der beschriebenen Zellen doppelter Art ordnen sich sehr mannichfaltig an: sie ziehen entweder in radiärer Richtung oder parallel mit der Oberfläche des Organs, bilden Krümmungen $\mathfrak{u}$. s. w. Demnach sind die Bilder unschwer zu verstehen, die sich auf Schnitten aus dieser Stelle der Marksubstanz darbieten. Die Zellreihen schneiden sich in verschiedenen Richtungen: in longitudinaler, schräger, transversaler u. s. w. In jeder Bindegewebsmasche liegt entweder eine Reihe von Zellen, und in diesem Falle grenzt jede der letzteren mit ihren Enden beiderseits an Bindegewebe, oder es befinden sich in einer Masche zwei solche Reihen; in diesem Falle liegen die Kerne nicht im Centrum der Zellen, sondern in den der Mitte der betreffenden Maschen zugekehrten Enden, so dass auf Längsschnitten die Kerne in zwei Reihen der Axenlinie entlang angeordnet erscheinen.

Bemerkenswerth ist das Vermögen der Zellen der Marksubstanz in Lösungen der Chromsäure und ihrer Salze, braune Fär- 
bung anzunehmen. Am besten kommt diese Färbung zu Stande, wenn man die Marksubstanz der Einwirkung dieser Reagentien nicht zu lange unterwirft - nicht mehr als 1-3 Tage. In solchem Falle werden die Zellen der Rindensubstanz dureh doppeltehromsaures Kali gar nicht gefärbt, und der Unterschied zwischen Rinden- und Marksubstanz tritt sowohl makro- als mikroskopisch ausserordentlich dentlich hervor: neben den hellen Zellen der Rindensubstanz liegen die braunen Zellen der Marksubstanz. Uebergangsformen zwischen den Zellen beider Art sind bei Säugethieren nicht vorhanden. Das Vermögen der Zellen der Marksubstanz mit Chrompräparaten sich zu färben, hängt höchst wahrscheinlich von einem besonderen Stoffe $a b$, der in ihnen enthalten ist und der sich, wie v. Brnnn gezeigt hat, in Alkohol löst, wobei dieser eine röthliche Färbung annimmt. Es ist jedoch zu bemerken, dass die Reaction mit Chromsäure nur an vollkommen frischen Nebennieren, die den soeben getödteten Thieren entnommen sind, gut gelingt; bei längerer Aufbewahrung des Organs scheint der die Färbung bedingende Stoff zu zerfallen oder einen anderen chemischen Charakter zu erlangen, so dass die Zellen fast ganz die Fähigkeit sich färben, verlieren. Hierauf beruht höchst wahrscheinlich der Umstand, dass die Marksubstanz der Nebennieren des Menschen, die der Leiche entnommen sind, fast gar nicht die Reaction mit Chromsüure hervortreten lässt. Nicht alle Theile der Marksubstanz färben sich mit gleicher Intensitäit, was wohl von einem verschiedenen Gehalt an den erwähnten Stoffen abhängen mag. Gewöhnlich wechseln auf Schnitten Zellreihen mit intensiverer Färbung mit Zellreihen ab, deren Färbung eine schwächere ist; dabei ist der Umstand bemerkenswerth, dass die dunkleren Zellen oder mit anderen Worten diejenigen, die in grösserer Menge den genannten Stoff enthalten, viel besser conservirt sind als die hellen Zellen. Demnach scheint die Beständigkeit der Zellen an die Gegenwart jenes Stoffes' gebunden zu sein. Weil dieser Stoff, wie oben angegeben, in nicht frischen Nebennieren verschwindet oder einen anderen chemischen Charakter annimmt, so beruht höchst wahrscheinlich darauf der schnelle Zerfall der Marksubstanz in den Nebennieren der Leichen von Thieren und Menschen. In Uebereinstimmung mit Henle und im Gegensatz zu der Behauptung v. Brunn's muss ich constatiren, dass die Zellkerne auch an der Färbung sich betheiligen und sich selbst intensiver färben als das Protoplasma. 
Ein Beitrag zur mikroskop. Anatomie der Nebennieren bei Säugethieren. 287

Die Marksubstanz nimmt beim Pferde einen geringeren Raum im Verhältniss zur Rindensubstanz ein als bei den übrigen vorbin genannten Thieren. Gebaut ist dieselbe im Allgemeinen ebenso, nur sind die Zellen kleiner als beim Rinde und beim Schweine und haben meist eine polygonale Gestalt. Beim Hunde, beim Kaninchen, bei der Katze und bei der Ratte sind die Bindegewebsmaschen meist rund und demnach liegen die Zellen nicht in Reihen, sondern ordnen sich den scharf contourirten Häufchen an, die an Drüsenbläschen, z. B. der Speicheldrüsen, erinnern.

In der oben beschriebenen Weise ist der grösste Theil der Marksubstanz eingerichtet. Stellenweise jedoch - am deutlichsten zeigt sich dies beim Rinde ausgeprägt - kommen Bezirke hervor, die eine im höchsten Grade eigenthümliche und interessante Struktur besitzen. Diese Stellen liegen beim Rinde meist in den centralen Partien der Marksubstanz, d. h. dort, wo das Bindegewebe gut entwickelt ist. Auf Schnitten aus diesen Bezirken sieht man, dass das Bindegewebe runde, ovale oder längliche Räume bildet. Die innere Oberfläche dieser Räume ist von einer Schicht niedriger, cubischer Zellen ausgekleidet, die eng bei einander liegen und sich in Müller'scher Flüssigkeit gar nicht färben. Der ganze innere Theil oder das Centrum eines solchen Raumes ist vollkommen von echten Markzellen ausgefüllt, die in Müller'scher Flüssigkeit sich stark brann färben. Diese Zellen liegen dicht bei einander und haben in Folge dessen eine polygonale Form. Demnach bildet hier das Bindegewebe allseitig geschlossene Bläschen oder Follikel, deren Wand von einer Reihe von Zellen ausgekleidet erscheint, die in M iuller'scher Flüssigkeit sich nicht fầrben, während ihr Binnenraum von echten Markzellen ausgefitllt ist. Diese letzteren werden durch eine Reihe heller Zellen vom Bindegewebe getrennt. Aehnliche Bläschen kommen gruppenweise vor, wobei die einen von ihnen einander dicht anliegen, so dass das Bindegewebe dazwischen fast unsichtbar wird, und die anderen isolirt angeordnet sind. In der beigefügten Figur 6 sind mehrere solche dicht einander anliegende Bläschen dargestellt. Bei $a$ sieht man einen Complex echter Markzellen, die durch Müller'sche Flüssigkeit tief gefärbt sind; $b$ ist eine einschichtige Reihe heller Zellen, die die Markzellen umgeben; bei $c$ erscheint diese Schicht von der Fläche durchschnitten, bei $d$ sind die Markzellen heransgefallen und nur die wandständigen hellen Zellen zurück- 
greblieben. Diese Reihe von Zelleu ist scharf vom umgebenden Gewebe geschieden. Stellenweise sieht man zwischen ibnen eine scharfe Linie, die den Durchschnitt einer das ganze Organ umhüllenden Membrana propria zu repräsentiren scheint. Von ihrer unzweifelhaften Existenz war ich jedoch nicht im Stande mich zu überzeugen.

Der Reichthum der Nebennieren an nervösen Elementen ist zu allen Zeiten den Forschern aufgefallen und daher wurde denselben in allen Arbeiten eine besondere Aufmerksamkeit zu Theil. Die Mehrzah! der Forscher nimmt an, dass die Nervenstämme ohne sich zu verzweigen die Rindensubstanz passiren und erst an der Grenze der Marksubstanz Aeste abzugeben beginnen, um in diesen letzteren einen dichten Plexus zu bilden. In der Beschreibung der Nervenzellen und Ganglien bestehen indessen erhebliche Differenzen. Einzelne Autoren stellen ihr Vorhandensein in den Nebennieren sogar vollkommen in Abrede. So sagt z. B. J.Arnold in seiner klassischen Abbandlung tiber die Nebennieren, dass in diesen gar keine Elemente vorkämen, die man als Nervenzellen ansprechen dürfte. Ecker findet sie nur in den Nebennieren des Pferdes. Andere Forscher, wie z. B. Leydig, Luschka, J. Meyer, vertheidigen eine vollkommen entgegengesetzte Anschauung. Nach ihrer Meinung hat die Mehrzahl der zelligen Elemente der Marksubstanz den Charakter von Ganglienzellen. Endlich glaubt eine dritte Reihe von Forschern, der Virchow ${ }^{17}$ ), Holm ${ }^{18}$ ), Moers, Grandry, Pfortner ${ }^{19}$, Rauber, Gottscha u. A. angehören, dass der grössere Theil der Marksubstanz aus specifischen zelligen Elementen bestehe, zwischen welchen entweder frei oder in die Bahn der Nervenstämme eingeschaltet, Nervenzellen liegen. Holm beschreibt ausser unzweifelhaften Nervenzellen noch Elemente anderer Art unter dem Namen: „Zellen von zweifelhaft nervöser Natur." Sie befinden sich in der Marksubstanz und sind in Gruppen verschiedener Grösse und Form angeordnet. Diese Zellen unterscheiden sich scharf sowohl durch ihre Form als durch ihre Anordnung von den Zellen der Marksubstanz. Durch ein Häufehen solcher Zellen tritt gewöhnlich ein Nervenstamn, der sich entweder in dem Häufchen verästelt oder das letztere umspinnt. Die Zellen sind oval mit abgestumpften Enden, mit grossem Kern und Kernkörperchen. Ihre wahrscheinliche Zugehörigkeit zum Nervensystem folgert Holm erstens aus der Loca- 
lität - sie liegen immer in der Nähe von Nerven - und zweitens aus dem Verhalten gegen Reagentien: gleich den unzweifelhaften Nervenzellen färben sie sich schnell mit Carmin, während die Zellen der Marksubstanz dies nur ziemlich langsam thun. Die Nervenbündel (nach Kölliker circa 30 für jede Drïse) treten an die Drüsen heran unter Bildung eines dichten Plexus. Aussen, unmittelbar dem Organ anliegend, befinden sich grosse Ganglien, auf deren Durchschnitt man mehr als 60 Nervenzellen zählen kann. Solche Ganglien fand ich bei allen von mir untersuchten Thieren. Sie sitzen nicht in der Kapsel selbst, wie es v. Brunn behauptet, sondern nach aussen von ihr, und obwohl sie derselben eng anliegen, sind sie jedoch mit ibr nur durch lockeres Bindegewebe verbunden, so dass sie bei der Anfertigung von Schnitten leicht herausfallen. Nachdem die Nervenbiundel die Kapsel durchsetzt haben, verlaufen sie ohne sich zu theilen durch die ganze Rindensubstanz bis dicht an die Marksubstanz; hier beginnen sie sich zu verästeln und bilden ein Netz aus Nervenbündeln verschiedener Dicke. Macht man einen Schnitt durch die Marksubstanz, so erscheinen die Nerven in allen Richtungen durchschnitten. Häufig, besonders in der Nebenniere des Rindes, sind neben ihnen besondere Gruppen von Zellen, die sich scharf von den Zellen der Marksubstanz unterscheiden, gelegen. Diese Gruppen umgeben entweder vollkommen je einen Nervenstamm, und dann befindet sich letzterer auf Querschnitten im Centrum einer Gruppe, oder sie liegen den Nerven nur von einer Seite an. Mitunter theilt sich der Nervenstamm in einem Häufchen solcher Zellen. Je feiner der Nervenstamm, um so kleiner werden die ihm anliegenden Zellgruppen, mit anderen Worten - mit der Verästelung der Nerven zerfallen auch die Zellcomplexe, die anfangs, $d . h$. in den peripheren Theilen der Marksubstanz, so umfangreich waren. Die Zellen haben eine eckige Form, sind scharf contourirt und liegen dicht einander an. In jeder Zelle befindet sich ein grosser Kern und in diesem ein Kernkörperchen. Nach den Abbildungen und nach der Beschreibung zu urtheilen, sind das eben die Zellen, die Holm Zellen von zweifelhaft nervöser Natur genannt hat. Sie haben aber nichts gemein mit wirklichen Nervenzellen und gar keine Aebnlichkeit mit solchen. Um über ihre wahre Natur in's Klare zu kommen, muss man einen Nervenstamm bis zu seinem Uebertritt aus der Marksubstanz in die Rindensubstanz verfolgen; dann siebt man, 
dass die fraglichen Zellgruppen, die den Nerven durch die ganze Marksubstanz begleiten, unmittelbar in die Rindensubstanz tibergehen. Dabei kann man sich ïberzeugen, dass sie sich von Zellen der Rindensubstanz gar nicht unterscheiden. Indem ein Nervenstamm aus der letzteren in die Marksubstanz übertritt, nimmt er einen gewissen Complex von Zellen mit sich, die anfangs, $d . h$. in den pheripheren Theilen der Marksubstanz, der Bindegewebsscheide des Nerven eng anliegen und denselben in Form ziemlich dicker Scheiden umhüllen, mit der fortschreitenden Theilung der Nerven aber* in kleinere Häufchen zerfallen. Diese Zellgruppen lassen sich, wie ich bereits erwähnt habe, längs der Bahrr der Nervenstämme verfolgen bis zu ihrer Verschmelzung mit der Rindensubstanz, so dass

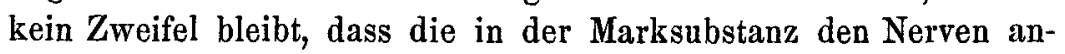
liegenden Zellgruppen aus Elementen der Rindensubstanz bestehen. Ausserdem besitzen die betreffenden Zellen dieselbe Form wie die Zellen der Rindensubstanz und verhalten sich ähnlich gegen Farbstoffe und chemische Reagentien. Durch Auspinselung kann man in jenen Gruppen ein feinstes bindegewebiges Reticulum nachweisen, ein ganz ähnliches Reticulum wie in der Rindensubstanz. Mithin ist es unzweifelhaft, dass die fraglichen Zellen Zellen der Rindensubstanz sind, auf deren Vorkommen in der Marksubstanz seit Arnold von vielen Autoren hingewiesen wird. Holm selbst findet eine Aehnlichkeit zwischen diesen Zellen und den Zellen der Rindensubstanz, ist aber trotzdem geneigt sie als dem Nervensystem angehörig zu betrachten. Gruppen von Zellen der Rindensubstanz in der Marksubstanz sind nur in den Nebennieren des Rindes, des Schafes und des Schweines deutlich zu sehen; bei den anderen Thieren kommen solche Gruppen entweder blos in dẹ am meisten peripher gelegenen Theilen der Marksubstanz vor oder sie kommen gar nicht vor.

Abgesehen von diesen Elementen, die mit Nervenzellen gar keine Aehnlichkeit haben und mit dem Nervensystem gar nichts zu thun haben, giebt es in der Marksubstanz der Nebennieren nun unzweifelhafte Nervenzellen. Zunächst ist die Thatsache festzuhalten, dass Ganglien und Ganglienzellen lediglich zur Marksubstanz in Beziehung stehen. In der Rindensubstanz kommen Nervenzellen nicht vor. Sie liegen entweder inmitten der Elemente der Marksubstanz oder an der Grenze der letzteren gegen die Rindensubstanz. In den oben beschriebenen, den Nebennieren von aussen 
Ein Beitrag zur mikroskop. Anatomie der Nebennieren bei Säugethieren. 291

anliegenden Ganglien kann man immer verschieden grosse Gruppen von Zellen finden, welche die Gestalt und alle Eigenschaften der Zellen der Marksubstanz aufweisen. Sie färben sich gleich den letzteren deutlich mit Salzen der Chromsäure. In anderen Fällen, zumal bei kleineren Thieren, erstreckt sich die Marksubstanz auf jener Seite, wo ein Ganglion sitzt, in Form eines Stranges durch die ganze Rindensubstanz und scheint mit dem Ganglion in Verbindung zu treten. Demnach sind diese ausserbalb gelegenen Ganglien gewissermaassen accessorische primitive Nebennieren, in welchen es lediglich nervöse Elemente und Zellen der Marksubstanz giebt. Nervenzellen fand ich in der Marksubstanz beim Menschen, beim Rinde, beim Pferde, beim Schafe, beim Schweine, beim Meerschweinchen, bei der Ratte und auch beim Kaninchen, gegenüber der Behauptung von Gottschau, dass es in der Marksubstanz der Nebennieren des Kaninchens keine Nervenzellen gäbe. Am zahlreichsten sind sie beim Mensehen, beim Rinde, beim Schafe und von kleineren Thieren beim Meerschweinchen. Die Nervenzellen sitzen entweder an den dicken Nervenstämmen oder sie liegen frei im Parenchym; in letzterem Falle sind sie.entweder in Gruppen angeordnet und bilden Ganglien oder sie liegen vereinzelt zwischen den zelligen Elementen der Marksubstanz. Eine solche Mannichfaltigkeit in der Anordnung der Zellen gilt sowohl in Bezug auf verschiedene Thiere als auch in Bezug auf ein und dasselbe Individuum. In den Nervenstämmen sitzen die Zellen entweder vereinzelt oder in Gruppen von je zwei bis sechs Zellen: Am häufigsten sitzen sie an den Theilungsstellen der grossen Nervenstämme. Die Zellen haben das Aussehen und alle Eigenschaften unzweifelhafter Nervenzellen. Ihr Protoplasma ist feinkörnig und enthält einen grossen bläschenförmigen hellen Kern mit deutlich sichtbarem Kernkörperchen. Neben einem Nerven, der unzweifelhafte Nervenzellen fübrt, liegen oft die oben beschriebenen Gruppen aus Zellen der Rindensubstanz. Nervenzellen, die nicht in der Bahn der grossen Nervenstämme sich befiuden, sind, wie bereits erwähnt, entweder zu Ganglien vereinigt oder sie liegen vereinzelt und isolirt; es verlaufen jedoch immer. Nervenfasern in der Nachbarschaft solcher Ganglien oder vereinzelter Zellen. Anlangend die Form, Grösse und das Vorkommen der Ganglien, so sind in dieser Beziehung bedentende Variationen vorhanden. Grossen Ganglien, welche auf dem Durch. 
schnitte 20 bis 60 Zellen haben, begegnete ich beim Menschen, beim Rinde, beim Schafe, beim Schweine und beim Meerschweinchen. Die Form solcher Ganglien ist rund oder oval. Ueber ihre Zahl und den Ort ihres Vorkommens lässt sich nichts Bestimmtes aussagen. Vor allen Dingen ist zu bemerken, dass ihnen gar kein bestimmter Ort zukommt; in der Mehrzahl der Fälle liegen sie in der Nähe der grossen Venen, d. h. näher nach dem Centrum der Marksubstanz. Ihre Zahl ist weder bei einem und denselben Individuum noch bei verschiedenen Thieren gleich. Am zahlreichsten sind sie beim Menschen und bei grösseren Thieren. Beim Meerschweinchen sieht man in der Marksubstanz ein grosses Ganglion, das auf dem Durchschnitt mehr als den dritten Theil der Marksubstanz einnimmt. Das Ganglion ist gewöhnlich von einer bindegewebigen Membran umhüllt, von der nach innen Fasern abgehen, welche die Zelleu von einander abgrenzen; ausserdem ist das Ganglion von zahlreichen Nerven und Blutgefässen durchsetzt. Um jede Zelle herum sieht man eine besondere kernhaltige Membran oder Kapsel. Beim Pferde ist die Zahl der Nervenzellen viel geringer als beim Rinde, und dieselben liegen hänfiger auf der Grenze zwischen Rinden- und Marksubstanz. Zuweilen sind diese Ganglienzellen von den oben beschriebenen fettig infiltrirten Zellen umgeben und enthält ihr Protoplasma Fettkörner, wie man dies auf Fig. 3 sehen kann.

Bisher wurde der Beziehung zwischen Nervenzellen und Zellen der Marksubstanz wenig Aufmerksamkeit geschenkt. Braun ${ }^{20}$ ) beschreibt bei Reptilien Uebergangsformen zwischen den Zellen beider Art und hält die Holm'schen Zellen für solche Uebergangsformen. J. Me yer ${ }^{21}$ ) beschreibt im System des Sympathicus, abgesehen von Theilen, die Alle als zum Nervensystem gehörig anerkennen, besondere Elemente, die sowohl zwischen wirklichen Nervenzellen sitzen als auch isolirt im Bindegewebe liegen. Diese Körper unterscheiden sich scharf von echten Nervenzellen. Ihre Form ist rund oder eckig. Sie enthalten eine verschiedene, unter Umständen eine grosse Anzahl von Kernen und Pigment. J. Meyer nennt diese Gebilde Kernnester oder Zellennester und schreibt ihnen eine grosse Rolle zu bezüglich der Entwickelung echter Nervenzellen. Indem Meyer sich der Meinung der Autoren anschliesst in Bezug auf die Möglichkeit einer Neubildung von Nervenzellen im Organismus, bestreitet er die Anuahme einer Neubildung 
Ein Beitrag zur mikroskop. Anatomie der Nebennieren bei Säugethieren. 293

dieser Zellen auf dem Wege einer Theilung der alten. Nach seiner Ansicht entstehen neue Nervenzellen aus den oben erwähnten Zellennestern; diese letzteren entstehen ihrerseits aus Blutkörperchen, die aus den Gefässen herausgetreten sind. Bei weiterer Untersuchung des sympathischen Systems bei niederen Thieren, wie z. B. beim Frosch, Triton, Salamander, gelangte er zu der Ueberzeugung, dass die sogenannten Nebennieren bei diesen Thieren ebenfalls dem Nervensysteme angehören. Dieselben enthalten: 1) Nerven, 2) Nervenzellen, 3) Kernnester, 4) besondere Zellen mit fettigem Inhalt, auf welchen eben die gelbliche Färbung des Ganglion zurückzuführen ist. Mit Rücksicht auf die übliche Eintheilung des Parenchyms der Nebennieren in eine Rinden- und eine Marksubstanz zählt Meyer zur ersteren die Zellen mit fettigem Inhalte und hält die Zellennester für Zellen der Marksubstanz. Die Nebennieren der höheren Thiere betrachtet er gleichfalls als zum Nervensysteme gehörig und meint, dass die Zellen der Marksubstanz bei diesen Thieren vorwiegend seine Zellennester repräsentiren. In einem Nachtrag führt Meyer Ausziuge aus einer Abhandlung von Stannius an, welcher annimmt, dass bei Fischen die Nervenzellen sich unter anderem auch in den Nebennieren entwickeln. In diesen Körpern soll man Bilder ihrer Entstehung und ihres Todes beobachten können. Mithin nehmen sowohl Braun als auch J. Meyer und Stannius eine Neubildung von Nervenzellen in den Nebennieren an. Indem ich mich zu den Nebennieren der Saiugethiere wende, muss ich sagen, dass ich hier nichts dergleichen fand, was J. Meyer unter dem Namen von Kern- oder Zellennestern beschrejbt. Uebergangsformen zwischen Zellen der Marksubstanz und Nervenzellen, wie es Braun beschreibt, habe ich auch nicht gefunden. Demnach vermag ich mit Bezug anf die Neubildung von Nervenzellen in den Nebennieren der Säugethiere nichts Bestimmtes auszusagen. Andererseits kommt man in die Lage bei näherem Studium der Marksubstanz Bilder zu seben, die anf eine regressive Metamorphose der Nervenzellen hinzuweisen scheinen, eine Metamorphose, die ibrerseits von dem Einfluss abzuhängen scheint, den die Zellen der Marksubstanz auf die Nervenzellen ausiiben. Ein Theil der Nervenzellen - diejenigen, die im Bindegewebe liegen und weiter entfernt von den Markzellen hat ein ganz normales Aussehen; das Protoplasma ist hell, der Kern tritt deutlich hervor, und der Zellkörper füllt die Kapsel 
vollkommen aus. Die anderen Nervenzellen - diejenigen, die inmitten der Zellen der Marksubstanz liegen, unterscheiden sich von den soeben beschriebenen. Die einen scheinen noch von normalem Aussehen zu sein und werden nur dicht von Zellen der Marksubstanz allseitig umlagert. In anderen Fällen dringen die letzteren in den Kapselraum ein und ordnen sich zwisehen der Kapsel und der Nervenzelle an, so dass diese von jener durch eine Reihe von Markzellen geschieden ist; in Folge dessen treten im Protoplasma der Nervenzelle Einkerbungen auf, und die ganze Zelle erhält ein sternförmiges Aussehen. In den weiteren Stadien der regressiven Metamorphose dringen immer mehr und mehr Zellen in die Kapsel der Nervenzelle ein und drücken die letztere zusammen; das Protoplasma derselben wird körniger und nimmt besser Farbstoffe an, der Kern wird undeutlich. Schliesslich kann man solchen Bildern begegnen, wo Markzellen die Kapsel vollkommen ausfüllen und wo von der Nervenzelle nur ein kleines Klümpchen von unregelmässiger Form und ohne Kern zurückgeblieben ist. Kurz, die Bilder machen einen solchen Eindruck, als ob die Nervenzellen infolge des auf sie ausgeiibten Druckes von Seiten der Markzellen zu Grunde gingen. Zum Zwecke der Beobachtung ähnlicher Bilder sind selbstverständlich solche Objecte zu benutzen, wo der Unterschied zwisehen specifischen Zellen der Marksubstanz und anderen zelligen Elementen deutlich zu sehen ist, d. h. es sind Nebennieren zu benutzen, die vorher mit doppeltehromsaurem Kali behandelt waren. Jene Bilder des Unterganges von Nervenzellen habe ich nicht nur bei erwachsenen Individuen, sondern auch bei jungen, wie z. B. in den Nebennieren von Kälbern, beobachtet. Die beigegebene Figur 7 stellt mehrere Nervenzellen in verschiedenen Stadien der regressiven Metamorphose dar. Bei a liegt zwischen einer Nervenzelle und ihrer Kapsel eine Reihe von Markzellen, bei $c$ füllen die letzteren die Kapsel fast vollkommen aus and von der Nervenzelle ist nur ein Klümpchen zurückgeblieben.

\section{Erklärung der Abbildungen auf Tafel XIII.}

Fig. 1. Aus der Rindensubstanz der Nebennieren des Schweines, $a=$ die Parenchymzellen, $b=$ das engmaschige Bindegewebsnetz.

Fig. 2. Aus der Rindensubstanz des Pfrrdes $c=$ Blutcapillaren. 
Ein Beitrag zur mikroskop. Anatomie der Nebennieren bei Säugethieren. 295

Fig. 3. Ans der Nebenniere des Pferdes. Die Grenze zwischen Rindenund Marksubstanz. $m=$ Marksubstanzzellen, $f=$ Gruppen fettig infiltrirter Zellen, $g=$ Ganglienzellen, welche Fetttröpfchen in ihrem Protoplasma enthalten.

Fig. 4. Aus der Nebenniere des Kaninchens. Die Grenze zwischen Rindenund Marksubstanz. Die Bezeichnungen wie oben.

Fig. 5. Zellenreihen aus der Marksubstanz des Rindes. $a=$ die bläschenförmigen Zellen, welche sich in Müller'scher Flüssigkeit schwach färben, $b=$ die oylindrischen Zellen, welche sich tief in Müller'scher Flüssigkeit färben.

Fig. 6. Aus der Marksubstanz des Rindes. $a=$ Marksubstanzzellen, $b=$ Schicht cubischer Zellen, die sich in Müller'scher Flüssigkeit nicht färben. Bei $c$ ist diese Schicht von der Fläche zu sehen. $n=$ Querschnitt von Nervenbündeln.

Fig. 7. Aus der Marksubstanz des Rindes. $g g g=$ Ganglienzellen, $m=$ Marksubstanzzellen, $r=$ Haufen von Rindensubstanzzellen in der Mark: substanz. $A=$ Ganglienzelle, noch gut erhalten. $B=$ Rest einer geschrumpften Ganglienzelle, umgeben von Marksubstanzzellen.

\section{Literatur.}

1) Henle, Handbuch der systematischen Anatomie des Menschen. Bd. II. 1873.

2) A. Ecker, Der feinere Bau der Nebenniere beim Menschen und den vier Wirbelthierklassen. 1846.

3) A. Ecker, Blutgefässdrüsen. Handwörterbuch d. Physiologie. Bd. IV. 1853.

4) Köllik er, Gewebelehre. 1867 .

5) Gerlach, Handbuch der allgemeinen und speciellen Gewebelehre des menschlichen Körpers. Wien. 1860.

6) Luschka, Die Anatomie des Menschen. Bd. II. Abth. I. 1863.

- Der Hirnanhang und die Steissdrüse des Menschen. Berlin. 1860.

7) Leydig, Lehrbuch der Histologie des Menschen und der Thiere. 1857.

8) Grandry, M., Mémoire sur la structure de la capsule surrénal de l'homme etc. Journal de l'anatomie et de sa phys. normales et pathol. 1867.

9) Arnold, J., Ein Beitrag zur feineren Structur der Nebenniere. Virchow's Archiv. Bd. XXXV. 1866.

10) Möers, A., Ueber den feineren Bau der Nebenniere. Virchow's Archiv. Bd. XXIX. 1864.

11) v. Brunn, A., Archiv f. mikroskop. Anatomie. Bd. VIII. 1872.

12) Joesten, Archiv der Heilkunde. Bd. V. 1864. 
13) Eberth, Die Nebennieren. Stricker's Handbuch. 1870.

14) Räuber, H., Zur feineren Structur der Nebennieren. Inaug.-Diss. Berlin.. 1881.

15) Gottschau, Ueber die Nebennieren der Säugethiere etc. Sitzungsbericht d. phys.-medic. Gesellsch. zu Würzburg. 1882.

16) Henle, Ueber das Gewebe der Nebenniere und der Hypophyse. Zeitschrift f. rat. Med. 3. Reihe. Bd. XXIV. 1865.

17) Virchow, Zur Chemie d. Nebenniere. Virchow's Archiv. XII. 1857.

18) Holm, Ueber die nervösen Elemente in den Nebennieren. Wiener Sitzungsberichte. Bd. LIII. Abth. I. 1866.

19) P fortner, Untersuchungen über das Ganglion intercaroticum und die Nebenniere. Zeitschrift f. rat. Med. Bd. XXXV. 1866.

20) Braun, M., Ueber Bau und Entwicklung der Nebenniere bei Reptilien. Zool. Anzeiger. II. Jahrg. 1879.

- Bau und Entwicklung der Nebennieren bei Reptilien. Arbeiten d. Zool. Instituts zu Würzburg. Bd. V.

21) Mayer, J., Wiener Sitzungsberichte. Bd. LXVI. Abth. II.

\section{Zur Anatomie und Physiologie der Leuchtorgane mexikanischer Cucuyo's.}

Von

Carl Heinemann in Vera-Cruz.

Vor längerer Zeit habe ich im 8. Bande dieses Archivs Beitrïge zur Kenntniss der Leuchtorgane der bei Vera Cruz volkommenden Leuchtkäfer veröffentlicht, welche sich wesentlich nur auf die sogenannten Cucuyo's (spanischer Name für die leuchtenden Elateren des tropischen Amerikas) bezogen. Obgleich eine baldige Fortsetzung jener nur allzu liackenhaften Untersuchungen in Aussicht gestellt worden war, blieben dieselben dennoch bis vor 4 Jahren ruhen, wo ich sie mit erneutem Eifer aufnahm und während vier Flugzeiten fortführte. Wenn ich auch jetzt nur wieder Fragmente liefere, so bitte ich den Leser $z u$ bedenken, dass die folgende Mittheilung die Frucht der oft knapp gemessenen Stunden der Erholung von meiner Thätigkeit als praktischer Arzt ist, und dass ich kaum über die einfachsten Apparate, geschweige 